\title{
Duality of boundary value problems and braneworld action in curved brane models
}

\author{
A. O. Barvinsky ${ }^{\dagger}$ and D. V. Nesterov* \\ November 12, 2018 \\ Theory Department, Lebedev Physics Institute, Leninsky Prospect 53, Moscow 11999, \\ Russia
}

\begin{abstract}
Braneworld effective action is constructed by two different methods based respectively on the Dirichlet and Neumann boundary value problems. The equivalence of these methods is shown due to nontrivial duality relations between special boundary operators of these two problems. Previously known braneworld action algorithms in two-brane Randall-Sundrum model are generalized to curved branes with deSitter and Anti-deSitter geometries.
\end{abstract}

†e-mail: barvin@lpi.ru

*e-mail: nesterov@lpi.ru

\section{Introduction}

Rapidly developing theory of braneworld phenomena [1, 2, 3] requires new efficient methods of their description. These methods incorporate together with the well-known old formalisms, like the effective action approach, special new features associated with bulk/brane (boundary) ingredients characteristic of the braneworld scenarios. In this paper we want to focus our attention at the peculiarities of the Dirichlet and Neumann boundary conditions in braneworld setup, the way they arise in the course of calculating braneworld effective action and, in particular, at a sort of duality relation between these problems. This relation manifests itself in the equality of special boundary operators originating from Dirichlet and Neumann boundary value problems and serving as a kernel of the braneworld effective action in the approximation quadratic in fields.

Braneworld effective action implicitly incorporates the dynamics of the fields in the bulk and explicitly features the boundary fields (being the functional of those) which, in braneworld scheme, are directly observable by the observer living on the brane. Such action, on the one hand, arises as a result of integrating out the bulk fields subject to boundary (brane) fields and, on the other hand, generates effective equations of motion for 
the latter. This situation obviously suggests two strategies of calculating the braneworld action. One strategy consists in its direct calculation and, in the tree-level approximation, reduces to solving the equations of motion in the bulk subject to given boundary values - brane fields - and substituting the result into the fundamental bulk action. Thus, this strategy involves the Dirichlet problem. Another strategy consists in recovering the braneworld effective action from effective equations of motion for brane fields. Since the latter incorporate well known Israel junction conditions on branes [四], this method relies on the Neumann boundary value problem. The consistency of these two methods, which obviously should lead to one and the same result, is far from being explicit. Here we show that they really match in view of a nontrivial relation between nonlocal brane operators arising as restriction of (properly differentiated) kernels of Dirichlet and Neumann Green's functions to the boundaries/branes. As a byproduct of the Dirichlet setup we derive the answer for the braneworld effective action in models with curved branes of deSitter and Anti-deSitter geometries.

Curved brane models have been considered in many papers their very incomplete list consisting of [5, 6, 7, 8, 9, 10]. In contrast to the results of these works we concentrate on two-brane models with independent metric configurations on their branes. This is done along the lines of the previous papers [13, 14 where the weak field (quadratic in perturbations) approximation for braneworld action was obtained on the background of flat branes. In distinction from [7, 9, 10] we obtain the nonlocal action nontrivially intertwining metric fields on both branes. The interest in such a construction follows from the attempts of describing off-shell phenomena in braneworld physics, solving the hierarchy and cosmological constant problems, generating inflation in braneworld scenarios [11, 13], etc.

The paper is organized as follows. In Sect.2 we define the braneworld effective action and sketch its calculation within the perturbation expansion with Dirichlet boundary conditions on branes. In Sect.3 we apply this technique to the two-brane Randall-Sundrum model 12 to find its action in the approximation quadratic in the perturbations of metric

fields on branes. This result generalizes the algorithms of the works [13, 14 to curved branes of deSitter and Anti-deSitter geometries. Its derivation is based on the technique of linearized invariants of the diffeomorphism gauge transformations intensively used in the theory of cosmological perturbations [15, 16, 17]. Sect.4 gives a review of the alternative calculation [14 of the same result, based on its recovery from the effective equations of motion. In Sect.5 we show the equality of the two manifestly different quadratic forms of the action by proving that their kernels are equal to one another - the main duality relation between specific operators of the Dirichlet and Neumann boundary value problems. Finally, in concluding section we discuss possible implications of the obtained results in braneworld scenarios with curved (inflating) branes.

\section{Braneworld effective action}

We will calculate the braneworld effective action defined by the procedure that was discussed in much detail in [14]. In contrast to the conventional Kaluza-Klein reduction, usually used for the construction of the effective action [18, 19], our action is a functional of 
the fields that have direct physical and geometrical interpretation. They coincide with the restriction $\phi$ of the fundamental bulk fields $\Phi$ to the branes, they are directly observable by observers living on these branes, and serve as boundary conditions for $\Phi$.

To be more precise, let $\Phi$ be a set of fundamental dynamical fields on $(d+1)$ dimensional spacetime $\mathcal{M}$ (bulk) with the full boundary $\partial \mathcal{M}$. In $\partial \mathcal{M}$ we shall include not only the "outer" boundary (like asymptotic domains of the bulk spacetime) but also the branes - timelike $d$-dimensional surfaces embedded into $\mathcal{M}$ and enumerated by the index $i, \partial \mathcal{M}=\bigcup_{i} \partial \mathcal{M}_{i}$. Boundary values of $\Phi$ on $\partial \mathcal{M}_{i}$ we denote by $\phi_{i},\left.\Phi\right|_{\partial \mathcal{M}_{i}}=\phi_{i}$, and call them brane (or induced) fields. In addition to the bulk $(d+1)$-fields there are usually $d$-dimensional fields $\varphi$ which, by definition, live only on branes and which will be in what follows denoted as matter fields'.

Thus, the full $(d+1)$-dimensional action is

$$
\mathbf{S}[\Phi, \varphi]=\mathbf{S}^{(d+1)}[\Phi]+S^{\mathrm{mat}}[\phi, \varphi],
$$

where the first term denotes the bulk part depending only on $\Phi$, while the second term represents the matter action which depends only on induced (brane) $\phi$ and matter fields $\varphi$. For brevity we omit the index $i$ of brane and matter fields, so that $(\phi, \varphi)$ denotes the collection of fields $\left(\phi_{i}, \varphi_{i}\right)$ associated with all the branes.

Braneworld effective action is the result of integrating out the bulk fields subject to given values of brane fields

$$
\exp \left(i S^{\mathrm{eff}}[\phi, \varphi]\right)=\left.\int D \Phi \exp (i \mathbf{S}[\Phi, \varphi])\right|_{\Phi(\partial \mathcal{M})=\phi} .
$$

Since the action of matter fields enters (四) additively and its arguments are not integrated over, it continues entering $S^{\text {eff }}[\phi, \varphi]$. The rest of the latter is highly nontrivial, because it accumulates the result of the functional integration. When this integration is done within $\hbar$-expansion the result reads

$$
S^{\mathrm{eff}}[\phi, \varphi]=S_{d}[\phi]+S^{\mathrm{loop}}[\phi]+S^{\mathrm{mat}}[\phi, \varphi]
$$

where the tree-level part

$$
S_{d}[\phi]=\mathbf{S}^{(d+1)}[\Phi[\phi]]
$$

is a result of substituting in the classical bulk action the solution $\Phi[\phi]$ of the following Dirichlet boundary value problem

$$
\left\{\begin{array}{l}
\frac{\delta \mathbf{S}^{(d+1)}}{\delta \Phi}=0, \\
\left.\Phi\right|_{\partial \mathcal{M}}=\phi .
\end{array}\right.
$$

Also, $S^{\text {loop }}[\phi]$ represents the loop part, of order $\hbar$, which we shall disregard in what follows.

\footnotetext{
${ }^{1}$ Usually the role of bulk fields is played by the bulk spacetime metric, because string theory motivated braneworld framework does not admit the propagation of gauge (matter) fields in the bulk [2].
} 
When considering the full nonlinear theory (1) it is generically impossible to obtain the tree-level term (4) as an exact explicit functional of induced fields $\phi$. Instead, one can develop a perturbation theory for (4) in powers of the perturbation of boundary conditions

$$
\phi=\phi^{0}+h
$$

on the background of some configuration $\phi^{0}$ at which the effective action is exactly calculable (or represents an irrelevant constant). This perturbation induces the perturbation $H[h]$ of the solution of the boundary problem in the bulk (5)

$$
\Phi[\phi]=\Phi^{0}+H[h],
$$

where $\Phi^{0}=\Phi\left[\phi^{0}\right]$ is the bulk background solving the classical equations of motion with background boundary conditions on branes $\phi=\phi^{0}$, which is also supposed to be explicitly known.

From (5) and (17) it follows that up to $O\left(h^{2}\right)$-terms, the bulk perturbation $H\left[\phi^{0}, h\right]$ satisfies the following linear Dirichlet problem:

$$
\left\{\begin{array}{l}
\hat{\mathbf{F}} H=0, \\
\left.H\right|_{\partial \mathcal{M}}=h
\end{array}\right.
$$

where $\hat{\mathbf{F}}$ is the operator of small field disturbances in the theory with the action (1). Since we assume that its Lagrangian contains at most first-order derivatives, $\hat{\mathbf{F}}$ is a second-order differential operator with the kernel in the space of $(d+1)$-dimensional bulk coordinates $X$ calculated on the background $\Phi^{0}$

$$
\hat{\mathbf{F}} \delta\left(X, X^{\prime}\right)=\left.\frac{\delta^{2} \mathbf{S}^{(d+1)}[\Phi]}{\delta \Phi(X) \delta \Phi\left(X^{\prime}\right)}\right|_{\Phi=\Phi^{0}} .
$$

As any other second-order differential operator, $\hat{\mathbf{F}}$ satisfies the Wronskian relation following from integration by parts in the equation

$$
\int_{\mathcal{M}} d V\left(\Phi_{1}\left(\hat{\mathbf{F}} \Phi_{2}\right)-\left(\hat{\mathbf{F}} \Phi_{1}\right) \Phi_{2}\right)=-\int_{\partial \mathcal{M}} d S\left(\Phi_{1}\left(\hat{W} \Phi_{2}\right)-\left(\hat{W} \Phi_{1}\right) \Phi_{2}\right)
$$

for arbitrary $\Phi$ and $\Psi$, for arbitrary test functions $\Phi_{1}$ and $\Phi_{2}$. Here $d V$ is the bulk integration measure and $d S$ is the measure on the boundary, and we shall call $\hat{W}$ the Wronskian operator. It is of the first order in derivatives and, for regular situations, it necessarily contains the derivative normal to the boundary. Obviously, this operator is defined by the relation (10) only up to addition of an arbitrary selfadjoint operator on the boundary. It can be specified uniquely by demanding that

$$
\int_{\mathcal{M}} d V \Phi_{1} \overleftrightarrow{\mathbf{F}} \Phi_{2}=\int_{\mathcal{M}} d V \Phi_{1}\left(\hat{\mathbf{F}} \Phi_{2}\right)+\int_{\partial \mathcal{M}} d S \Phi_{1}\left(\hat{W} \Phi_{2}\right)
$$

where the notation $\overleftrightarrow{\mathbf{F}}$ implies that the derivatives of $\hat{\mathbf{F}}$ are acting (in the sense of integration by parts) so that the left-hand side represents the form bilinear in first order 
derivatives. For $\Phi_{2}=\Phi_{1}$ the integrand of the left-hand side is just the Lagrangian quadratic in the field and its first-order derivatives. In this case the operator $\hat{W}$ can also be obtained in terms of the canonical momentum conjugated to the field relative to "time" normally flowing to the boundary [20].

This Wronskian operator allows one to formulate in a closed form the solution of the Dirichlet problem (8) given the Green's function of this problem satisfying

$$
\left\{\begin{array}{l}
\hat{\mathbf{F}} \mathbf{G}_{D}\left(X, X^{\prime}\right)=\delta\left(X, X^{\prime}\right) \\
\left.\mathbf{G}_{D}\left(X, X^{\prime}\right)\right|_{X \in \partial \mathcal{M}}=0
\end{array}\right.
$$

This solution reads as the following integral over the boundary $\partial \mathcal{M}$

$$
\Phi(X)=-\int_{\partial \mathcal{M}} d S^{\prime}\left[\mathbf{G}_{D}\left(X, X^{\prime}\right) \overleftarrow{W^{\prime}}\right]_{X^{\prime}=X\left(x^{\prime}\right)} h\left(x^{\prime}\right)
$$

Here $X=X(x)$ denotes the embedding of the boundary $\partial \mathcal{M}$ parameterized by internal coordinates $x$ into the bulk with coordinates $X, d S^{\prime}=d S\left(x^{\prime}\right)$ is the surface integration element at $x^{\prime}$ and the arrow over $W^{\prime}$ indicates that the derivatives of the primed Wronskian operator act to the left on the second (primed) argument of the Green's function.

The expansion of the action in the field perturbation (7) begins with

$$
S_{d}[\phi]=\mathbf{S}^{(d+1)}\left[\Phi^{0}\right]+\frac{1}{2} \int_{\mathcal{M}} d V H \overleftrightarrow{\mathbf{F}} H+O\left[h^{3}\right],
$$

because the linear term identically vanishes in virtue of classical equations of motion for $\Phi^{0}$ and, what is important, in virtue of the Israel matching conditions on branes, which annihilate the linear surface terms on boundaries even despite the fact that $\left.H\right|_{\partial \mathcal{M}}=h \neq 0$. Note that the quadratic part of this expansion is built with the aid of the operator $\stackrel{\leftrightarrow}{\mathbf{F}}$ symmetrically acting on both fields - the consequence of the fact that the initial action did not contain second-order derivatives. Therefore, using the relation (11) and taking into account linear equations of motion for perturbations (8) one finds that the bulk part of the quadratic term vanishes and the quadratic approximation for the braneworld effective action reduces to the surface term?

$$
S_{d}[\phi]=\mathbf{S}^{(d+1)}\left[\Phi^{0}\right]+\frac{1}{2} \int_{\partial \mathcal{M}} d S H(\hat{W} H) .
$$

Further, a substitution of (13) in this expression gives the general answer for the quadratic action

$$
S_{d}^{(2)}[h]=-\frac{1}{2} \int_{\partial \mathcal{M}} d S \int_{\partial \mathcal{M}} d S^{\prime} h(x) \overrightarrow{\mathrm{W}} \mathbf{G}_{D} \overleftarrow{\mathrm{W}}\left(x, x^{\prime}\right) h\left(x^{\prime}\right)
$$

\footnotetext{
${ }^{2}$ In what follows we restrict ourselves with the quadratic approximation and omit terms $O\left[h^{3}\right]$.
} 
where the kernel of the integral operation on the boundary $\vec{W} \mathbf{G}_{D} \overleftarrow{W}\left(x, x^{\prime}\right)$ is given by an obvious sequence of operations with the kernel of the Dirichlet Green's function - acting on it from both sides with Wronskian operators and restricting the result to the boundary with respect to both arguments

$$
\overrightarrow{\mathrm{W}} \mathbf{G}_{D} \overleftarrow{\mathrm{W}}\left(x, x^{\prime}\right)=\left[\vec{W} \mathbf{G}_{D}\left(X, X^{\prime}\right) \overleftarrow{W^{\prime}}\right]_{X=X(x), X^{\prime}=X\left(x^{\prime}\right)}
$$

As mentioned above, for the case of several branes or spacetime boundary consisting of several pieces $\partial \mathcal{M}=\bigcup_{i} \partial \mathcal{M}_{i}$ the boundary (brane) fields denote the collection $\phi=\phi^{i}$. Correspondingly the brane embedding functions and respective perturbations acquire the same index, $X=X_{i}(x), h=\left.h^{i} \equiv h\right|_{\partial \mathcal{M}_{i}}$, and the equations above imply the following obvious generalization

$$
\begin{aligned}
& \int_{\partial \mathcal{M}} d S \rightarrow \sum_{i} \int_{\partial \mathcal{M}_{i}} d S \\
& \overrightarrow{\mathrm{W}} \mathbf{G}_{D} \overleftarrow{W}\left(x, x^{\prime}\right) \rightarrow\left[\overrightarrow{\mathrm{W}} \mathbf{G}_{D} \overleftarrow{W}\right]_{i j}\left(x, x^{\prime}\right)=\left[\vec{W} \mathbf{G}_{D}\left(X, X^{\prime}\right) \overleftarrow{W^{\prime}}\right]_{X=X_{i}(x), X^{\prime}=X_{j}\left(x^{\prime}\right)}
\end{aligned}
$$

Under these replacements the expression (16) becomes a quadratic form with the nonlocal matrix-valued kernel (19).

\section{Two-brane Randall-Sundrum model}

Here we specify the construction of the above type on the example of the two-brane Randall-Sundrum model [12] with the action

$$
\begin{gathered}
\mathbf{S}[G, \varphi]=\int_{\mathbf{B} \times \mathrm{Z}_{2}} d^{d+1} X \sqrt{G}(R(G)-2 \Lambda)-2 \sum_{i} \int_{\mathbf{b}_{i}} d^{d} x \sqrt{g}[K]-\sum_{i} \int_{\mathbf{b}_{i}} d^{d} x \sqrt{g} \sigma_{i}+ \\
+\sum_{i} \int_{\mathbf{b}_{i}} d^{d} x \sqrt{g} L^{\operatorname{mat}}(g, \varphi, \partial \varphi)
\end{gathered}
$$

The orbifold symmetry implies that the $(d+1)$-dimensional integration runs over two identical copies of the bulk $\mathbf{B}$ which is bounded by two $d$-dimensional branes $\mathbf{b}_{i}, i= \pm$, that can be regarded as the boundaries of $\mathbf{B}$. Here $G=G_{A B}(X)(A=0,1, \ldots, d)$ is the bulk metric and $g=g_{\alpha \beta}^{ \pm}(x)(\alpha=0,1, \ldots, d-1)$ denotes the collection of induced metrics on $i= \pm$ branes, $[K]$ is a jump of the trace of the extrinsic curvature on the brane defined as $K=G^{A B} \nabla_{A} \mathbf{n}_{B}$, where $\nabla_{A}$ is a covariant $(d+1)$-dimensional derivative and $\mathbf{n}$ is an inward pointing normal. With this definition the normals on two sides of the brane are oppositely oriented and the extrinsic curvature jump $\left[K_{\alpha \beta}\right]$ actually equals the sum of the so-defined curvatures on both sides of the brane. The orbifold symmetry obviously implies $\int_{\mathbf{B} \times \mathbf{Z}_{2}}(\ldots)=2 \int_{\mathbf{B}}(\ldots)$ and $[K]=2 K$. Finally, $\Lambda$ is the bulk cosmological constant and $\sigma_{i}$ are brane tensions.

Our goal is to construct the perturbative braneworld effective action for such a system. The fundamental bulk field is the bulk metric $\Phi=G_{A B}(X)$, the role of brane field is played 
by $\phi=g_{\alpha \beta}^{ \pm}(x)$ - the induced metric on branes. The first three terms of (20) comprise the bulk part of the action and the last term is the action of matter fields located on branes?

We choose as the background $\Phi^{0}$ the well-known Randall-Sundrum solution in this model. In the setting generalized to the case of curved branes this solution arises as follows. Assume that there exists a coordinate system on the $(d+1)$-dimensional bulk $X^{A}=\left(x^{\alpha}, y\right)$ in which the background metric solving the vacuum Einstein equations has the form

$$
G_{A B}^{0}(X)=\left(\begin{array}{cc}
a^{2}(y) g_{\alpha \beta}(x) & 0 \\
0 & 1
\end{array}\right) .
$$

Two branes bounding the bulk manifold $\mathbf{B}$ are the hypersurfaces of constant $y$ located at $y=y_{+}$and $y=y_{-}$, so that $\mathbf{B}$ has a topology of $\mathbf{b} \times \mathbf{I}$ with $x^{\alpha}$ - the coordinates on $d$-dimensional $\mathbf{b}$ and $\mathbf{I}$ - the interval of the coordinate $y \in\left[y_{+}, y_{-}\right]$. In the wording of the previous section the boundary conditions $\phi^{0}$ for this bulk background $\Phi\left[\phi^{0}\right]$ are two induced metrics conformally equivalent to one another $g_{\alpha \beta}^{ \pm}=a^{2}\left(y_{ \pm}\right) g_{\alpha \beta}(x)$ and conformally related to some fixed metric $g_{\alpha \beta}(x)$. With the ansatz (21) this metric is restricted by the condition of constant scalar curvature. Indeed, from the $y y$-component of the vacuum Einstein equations in the bulk it follows that

$$
a^{-2}(y) R(g)-2 \Lambda=d(d-1)\left(\partial_{y} \ln a(y)\right)^{2},
$$

where $R(g)$ is the scalar curvature of $g_{\alpha \beta}(x)$. Therefore, only metrics with constant $R(g)$ independent of $x$ can satisfy this equation. They include, in particular, three maximally symmetric cases - deSitter $\left(d S^{d}\right)$, plane $\left(\mathbb{R}^{d-1,1}\right)$ and Anti-deSitter $\left(A d S^{d}\right)$ branes embedded into the Einstein space with the cosmological constant $\Lambda$. As in the Randall-Sundrum case we shall consider the case of anti-deSitter bulk with negative $\Lambda$

$$
\Lambda=-\frac{1}{2} d(d-1) k^{2}
$$

so that Eq.(22) gives the following profiles of the scale factor $a(y)$ for the foliation of the Anti-deSitter spacetime by the deSitter, flat and Anti-deSitter slices

$$
a^{2}(y)= \begin{cases}\frac{\mathcal{H}^{2}}{k^{2}} \sinh ^{2}(k y), & d S^{d} \\ \exp (-2 k y), & \mathbb{R}^{d-1,1} \\ \frac{\mathcal{H}^{2}}{k^{2}} \cosh ^{2}(k y), & A d S^{d}\end{cases}
$$

where the "Hubble" constant $\mathcal{H}$ is given by

$$
\mathcal{H}^{2}=\frac{|R(g)|}{d(d-1)} .
$$

\footnotetext{
${ }^{3}$ For reasons of convenience we include the brane tension terms into the bulk part of the action. As far as it concerns the Gibbons-Hawking term with the trace of extrinsic curvature, it should also be related to the bulk part because it makes by integration by parts the whole Einstein-Hilbert action quadratic in first-order derivatives.
} 
Among these three cases the original Randall-Sundrum model [12] corresponds to the foliation with flat slices. Two flat and empty branes coincide with the pair of such slices and serve as boundaries of a smooth piece of Anti-deSitter bulk at $y_{+} \leq y \leq y_{-}$. With the orbifold symmetry the piecewise smooth Anti-deSitter solution of vacuum Einstein equations satisfies the Israel junction conditions on flat branes when their tensions are opposite in sign and equalf

$$
\sigma_{+}=-\sigma_{-}=4 \sqrt{\frac{2(d-1)|\Lambda|}{d}} .
$$

Other cases of curved (deSitter and Anti-deSitter) branes correspond to different expressions for their tensions $\sigma_{i}=\sigma_{ \pm}$. For $Z_{2}$-orbifold interpolating between the two curved branes these tensions, when they satisfy Israel junction conditions, depend on the location of branes $y_{i}=y_{ \pm}$and read

$$
\sigma_{i}=-\left.4(d-1) \nabla_{\mathbf{n}} \ln a(y)\right|_{y_{i}},
$$

where we introduce the notation

$$
\nabla_{\mathbf{n}} \equiv \mathbf{n}^{A} \partial_{A}= \begin{cases}+\partial_{y}, & y=y_{+} \\ -\partial_{y}, & y=y_{-}\end{cases}
$$

for the inward pointing derivative normal to \pm -branes in the background metric (21). In order to match with the inward orientation of the normal vector (or location of the positive tension brane to the left of the negative tension one) we have to assume that the coordinate $y$ in (24) is negative for $d S$ and $A d S$ cases, $y_{+}<y_{-}<0$. This also guarantees that deSitter branes are not separated by particle horizon at which $a(y)=0$ [8, 23] and, thus, bound a causally connected bulk. Conversely, relations (27) can be viewed as the equations which for given bulk curvature parameter $k$ and two different brane tensions of opposite signs $\sigma_{+}, \sigma_{-}$determine the locations of branes at $y_{+}$and $y_{-}$.

Our goal now is to build, along the lines of Sect.2, the perturbation expansion for braneworld action on this background by perturbing the brane configurations like in (6) and studying the response to it in the bulk (7). The geometrical picture of the background solution above actually shows that a simple scheme of Sect.2 should be supplemented by a number of important modifications due to local diffeomorphism invariance of the theory. This invariance manifests itself in many facts. In particular, not all components of the bulk metric $G_{A B}(x, y)$ should be fixed as boundary conditions on the branes, but rather the induced metric coefficients $g_{\alpha \beta}$, whose number is less than that of $G_{A B}$ ?. Moreover, the induced metric coefficients are nontrivially related to $G_{A B}(x, y)$ depending on the

\footnotetext{
${ }^{4}$ For simplicity we dropped in Eq.(20) the gravitational coupling constant so that the action has the dimensionality of length to the power $d-1$ and the brane tensions have the dimensionality of inverse length.

${ }^{5}$ The distinguished status of the $G_{y A^{-}}$-components of the metric that should not be fixed at the boundary $y=$ const follows from the fact that they serve as Lagrange multipliers of constraints in the 'Hamiltonian' formalism associated with the $y$-'time' foliation of spacetime. This in turn follows from the local gauge invariance of the Einstein theory.
} 
embedding of boundaries in the bulk, this embedding being nontrivial after the inclusion of gravitational perturbations and depending on the gauge fixation of the latter.

These properties of the braneworld formalism were considered in much detail in a number of papers [14, 21, 22] where the location of branes in the bulk was described in the Gaussian normal coordinates, $G_{y y}=1, G_{y \mu}=0$, by an additional scalar field - the radion. Here we prefer to proceed in a more gauge invariant manner without explicitly fixing the coordinate system in the bulk. The only restriction on the choice of $(d+1)$-dimensional coordinates will be the requirement that the branes are located at constant values of the $y$-coordinate. With this gauge fixation freedom all metric elements are subject to nonvanishing perturbations $H_{A B}(x, y)$ which can be decomposed into $d$-dimensional scalar, vector and tensor parts $H_{A B}(x, y)=\left(H_{y y}(x, y), H_{y \alpha}(x, y), H_{\alpha \beta}(x, y)\right)$. The latter two in their turn can be decomposed in irreducible scalar, transverse and transverse-traceless components with respect to the fixed metric $g_{\alpha \beta}(x) \emptyset$. Thus we have

$$
\begin{aligned}
& H_{y y}(x, y)=2 \chi(x, y), \\
& H_{\alpha y}(x, y)=a^{2}(y)\left(v_{\alpha}(x, y)+\nabla_{\alpha} b(x, y)\right), \quad \nabla^{\beta} v_{\beta}(x, y)=0, \\
& H_{\alpha \beta}(x, y)=a^{2}(y)\left(\gamma_{\alpha \beta}(x, y)+2 \nabla_{(\alpha} f_{\beta}(x, y)+2 \psi(x, y) g_{\alpha \beta}(x)+2 \nabla_{\alpha} \nabla_{\beta} e(x, y)\right), \\
& \nabla^{\alpha} \gamma_{\alpha \beta}(x, y)=g^{\alpha \beta}(x) \gamma_{\alpha \beta}(x, y)=0, \quad \nabla^{\alpha} f_{\alpha}(x, y)=0 .
\end{aligned}
$$

Perturbations $H_{\alpha \beta}(x, y)$ are generated by the induced metric perturbations $h^{i}=h_{\alpha \beta}^{ \pm}(x)$

$$
h_{\alpha \beta}^{ \pm}(x)=a^{2}\left(y_{ \pm}\right)\left(\gamma_{\alpha \beta}(x)+2 \psi(x) g_{\alpha \beta}(x)+2 \nabla_{(\alpha} f_{\beta)}(x)+2 \nabla_{\alpha} \nabla_{\beta} e(x)\right)^{ \pm},
$$

as boundary conditions for the linearized Einstein equations in the bulk, $\psi\left(x, y_{ \pm}\right)=\psi^{ \pm}(x)$, $e\left(x, y_{ \pm}\right)=e^{ \pm}(x), f_{\alpha}\left(x, y_{ \pm}\right)=f_{\alpha}^{ \pm}(x), \gamma_{\alpha \beta}\left(x, y_{ \pm}\right)=\gamma_{\alpha \beta}^{ \pm}(x)$.

We know that the action (20) is invariant with respect to $(d+1)$-dimensional diffeomorphisms. Under their action with the vector field $\xi^{A}(x, y)=\left(\xi^{\alpha}(x, y), \xi^{y}(x, y)\right)$, whose $d$-dimensional part has a transverse-longitudinal decomposition in the metric $g_{\alpha \beta}(x)$

$$
\xi_{\alpha}(x, y) \equiv g_{\alpha \beta}(x) \xi^{\beta}(x, y)=\lambda_{\alpha}(x, y)+\nabla_{\alpha} \mu(x, y), \quad \nabla_{\alpha} \lambda^{\alpha}=0,
$$

irreducible components of (29)-(31) transform as

$$
\begin{aligned}
& \delta \chi=\partial_{y} \xi^{y}, \\
& \delta b=a^{-2} \xi^{y}+\partial_{y} \mu, \quad \delta v_{\alpha}=\partial_{y} \lambda_{\alpha}, \\
& \delta \psi=\psi+\left(\partial_{y} \ln a\right) \xi^{y}, \quad \delta e=\mu, \quad \delta f_{\alpha}(x, y)=\lambda_{\alpha}, \\
& \delta \gamma_{\alpha \beta}(x, y)=0 .
\end{aligned}
$$

It is easy to check that the following combinations are invariant under the action of these transformations [0]

$$
\begin{aligned}
& \mathcal{A}=\partial_{y} \psi+\psi \frac{a^{-2} R(g)}{d(d-1) \partial_{y} \ln a}-\chi \partial_{y} \ln a, \quad \mathcal{B}=\partial_{y} e-b+\psi \frac{a^{-2}}{\partial_{y} \ln a}, \\
& \mathcal{V}_{\alpha}=\partial_{y} f_{\alpha}-v_{\alpha} .
\end{aligned}
$$

\footnotetext{
${ }^{6}$ At least for small metric perturbations this is always possible, because the foliation by surfaces of constant y, Eq.(24), is regular in patches of the Anti-deSitter spacetime of interest.

${ }^{7}$ Note, that throughout the paper $d$-dimensional (Greek) indices are lowered and raised with the help of $g_{\alpha \beta}(x)$ and $\nabla_{\alpha}$ denotes the covariant derivative in the metric $g_{\alpha \beta}(x)$.
} 
The conformal part of the metric perturbation $\psi(x, y)$ is not invariant under general diffeomorphisms, but for the class of diffeomorphisms leaving the boundaries at constant values of the $y$-coordinate, $\xi^{y}\left(x, y_{ \pm}\right)=0$, boundary values $\psi^{ \pm}(x)$ are also not transformed.

One can check that the quadratic part of the action expresses in terms of these invariants, as it should be for the diffeomorphism invariant functional. Direct calculation shows that it decomposes in the sum of decoupled contributions of scalar, vector and graviton (transverse-traceless) sectors

$$
\mathbf{S}_{(2)}^{(d+1)}[H]=S_{\text {scal }}[\mathcal{A}, \mathcal{B}, \psi]+S_{\text {vect }}[\mathcal{V}]+S_{\text {grav }}[\gamma]
$$

which read?

$$
\begin{gathered}
S_{\text {scal }}[\mathcal{A}, \mathcal{B}, \psi]=2(d-1) \int_{\mathbf{B}} d V\left(d \mathcal{A}^{2}+2 \mathcal{A} \square \mathcal{B}-\frac{R(g)}{d(d-1)} \mathcal{B} \square \mathcal{B}\right) \\
\quad+2(d-1) \sum_{i} \int_{\mathbf{b}_{i}} d S \frac{a^{d-2}}{\nabla_{\mathbf{n}} \ln a} \psi\left(\square+\frac{R(g)}{d-1}\right) \psi, \\
S_{\text {vect }}[\mathcal{V}]=\int_{\mathbf{B}} d V \mathcal{V}_{\alpha}\left(\square+\frac{R(g)}{d}\right) \mathcal{V}^{\alpha}, \\
S_{\text {grav }}[\gamma]=\frac{1}{2} \int_{\mathbf{B}} d V\left(-\partial_{y} \gamma_{\alpha \beta} \partial_{y} \gamma^{\alpha \beta}+a^{-2} \gamma_{\alpha \beta} \square \gamma^{\alpha \beta}-\frac{2}{d(d-1)} a^{-2} R(g) \gamma_{\alpha \beta} \gamma^{\alpha \beta}\right) .
\end{gathered}
$$

Here

$$
\square=g^{\alpha \beta}(x) \nabla_{\alpha} \nabla \beta
$$

is the $d$-dimensional covariant d'Alembertian. Note that it is defined with respect to the $y$-independent metric $g_{\alpha \beta}(x)$ rather than the background metric $a^{2}(y) g_{\alpha \beta}(x)$. Also $d V$ is the covariant Riemannian measure in the bulk and $d S$ is the covariant measure on branes the latter again defined with respect to the auxiliary metric $g_{\alpha \beta}(x)$

$$
\begin{aligned}
& d V \equiv d^{d} x d y a^{d}(y) \sqrt{g(x)} \\
& d S \equiv d^{d} x \sqrt{g(x)}
\end{aligned}
$$

\subsection{Scalar and vector sectors}

Interestingly, the calculation of scalar and vector contributions in (39) does not require explicit solution of the bulk equations of motion. The reason is that the invariants $\mathcal{A}$, $\mathcal{B}$ and $\mathcal{V}_{\alpha}$ enter the Lagrangian without $y$-derivatives and, therefore, the same property holds for the variables $\chi, b$ and $v_{\alpha}$ algebraically entering (38). This in turn follows from the fact that these variables play the role of Lagrange multipliers of the nondynamical (in

\footnotetext{
${ }^{8} \mathrm{Up}$ to total derivatives in $x^{\alpha}$ directions. Derivatives in $y$-direction are kept, since they induce terms on branes.
} 
the extra "time" $y$ ) $y A$-components of Einstein equations. So, varying the action with respect to these Lagrange multipliers we have

$$
\begin{aligned}
& \frac{\delta \mathbf{S}_{(2)}^{(d+1)}}{\delta \chi}=-\left(\partial_{y} \ln a\right) \frac{\delta \mathbf{S}_{(2)}^{(d+1)}}{\delta \mathcal{A}}=-4 a^{d} \sqrt{g}(d-1)\left(\partial_{y} \ln a\right)(\square \mathcal{B}+d \cdot \mathcal{A})=0, \\
& \frac{\delta \mathbf{S}_{(2)}^{(d+1)}}{\delta b}=-\frac{\delta \mathbf{S}_{(2)}^{(d+1)}}{\delta \mathcal{B}}=-4 a^{d} \sqrt{g}(d-1) \square\left(\mathcal{A}-\frac{R(g)}{d(d-1)} \mathcal{B}\right)=0, \\
& \frac{\delta \mathbf{S}_{(2)}^{(d+1)}}{\delta v_{\alpha}}=-\frac{\delta \mathbf{S}_{(2)}^{(d+1)}}{\delta \mathcal{V}_{\alpha}}=-2 a^{d} \sqrt{g}\left(\square+\frac{R(g)}{d}\right) \mathcal{V}^{\alpha}=0 .
\end{aligned}
$$

In virtue of these equations the vector (41) and the bulk part of the scalar (40) contributions to the action vanish f and the result reduces to the brane term of (40).

Note that the scalar sector is completely local and of second order in derivatives. However, generally its kinetic terms are not positive definite. With the factors $\nabla_{\mathbf{n}} \ln a$ expressed in terms of tensions (27) it takes the form

$$
S_{\mathrm{scal}}\left[\psi^{ \pm}\right]=-8(d-1)^{2} \sum_{i= \pm} \int_{\mathbf{b}_{i}} d S \frac{a_{i}^{d-2}}{\sigma_{i}} \psi\left(\square+\frac{R(g)}{d-1}\right) \psi
$$

which clearly emphasizes its indefiniteness for two tensions of opposite signs, $\sigma_{+}>0$, $\sigma_{-}<0$. On the positive tension brane the conformal mode enters with the negative (ghost like) sign - exactly as in the Einstein theory ${ }^{\text {to }}$, while on the negative tension brane its sign is opposite. Only for Anti-deSitter branes with $y_{+}<0<y_{-}$both brane tensions can be positive - the case which we will not consider in detail.

For flat 4-dimensional branes, $R(g)=0, d=4$, this expression reduces to the result obtained in $[14]$.

\subsection{Graviton sector}

It is important that the tensor (transverse-traceless) sector decouples from the rest of variables not only in the action, but already at the level of solving the boundary value problem $-\gamma_{\alpha \beta}^{ \pm}(x)$ (and only $\gamma_{\alpha \beta}^{ \pm}(x)$ ) serve as boundary conditions for $\gamma_{\alpha \beta}(x, y)$. That is why the situation in this sector literally repeats the technique of Sect.2. The action in the graviton sector (42) after integration by parts reads

$$
S_{\text {grav }}[\gamma]=\frac{1}{2} \int_{\mathbf{B}} d V \gamma_{\alpha \beta}\left(\hat{\mathbf{F}} \gamma^{\alpha \beta}\right)+\frac{1}{2} \sum_{i} \int_{\mathbf{b}_{i}} d S a^{d} \gamma_{\alpha \beta} \nabla_{\mathbf{n}} \gamma^{\alpha \beta},
$$

\footnotetext{
${ }^{9} \mathrm{Up}$ to possible surface terms at the infinity $x^{\alpha}$-coordinates in the bulk which we assume vanishing because of vanishing sources everywhere except branes.

${ }^{10}$ This does not lead to physical instability because the conformal mode is not dynamically independent in view of constraints.

${ }^{11}$ To compare (47) with the equation (139) of [14] one should bear in mind that our conformal modes $\psi^{ \pm}$are rescaled relative to the conformal modes $\varphi^{ \pm}$in $[14], \varphi^{ \pm}=2 a_{ \pm}^{2} \psi^{ \pm}$.
} 
where

$$
\hat{\mathbf{F}}=a^{-d} \partial_{y} a^{d} \partial_{y}+a^{-2}\left(\square-\frac{2}{d(d-1)} R(g)\right)
$$

is a second order differential operator that governs the dynamics of gravitons in the bulk. Comparison of the equation (48) with (11) shows that the Wronskian operator $\hat{W}_{ \pm}$coincides with $a_{ \pm}^{d} \nabla_{\mathbf{n}}$ on respective branes. Therefore, the solution of the Dirichlet problem for transverse-traceless perturbations

$$
\left\{\begin{array}{l}
\hat{\mathbf{F}} \gamma_{\alpha \beta}(x, y)=0 \\
\left.\gamma_{\alpha \beta}(x, y)\right|_{\mathbf{b}_{ \pm}}=\gamma_{\alpha \beta}^{ \pm}(x)
\end{array}\right.
$$

in terms of the Dirichlet Green's function of the operator 49)

$$
\left\{\begin{array}{l}
\hat{\mathbf{F}} \mathbf{G}_{D}\left(x, y \mid x^{\prime}, y^{\prime}\right)=\delta\left(x, y \mid x^{\prime}, y^{\prime}\right) \\
\left.\mathbf{G}_{D}\left(x, y \mid x^{\prime}, y^{\prime}\right)\right|_{\mathbf{b}_{ \pm}}=0
\end{array}\right.
$$

where $\delta^{(d+1)}\left(x, y \mid x^{\prime}, y^{\prime}\right)=\delta^{d}\left(x-x^{\prime}\right) \delta\left(y-y^{\prime}\right) / a^{d}(y) \sqrt{g(x)}$ is the covariant $(d+1)$-dimensional $\delta$-function[ए], reads as

$$
\gamma(x, y)=-\sum_{i= \pm} \int_{\mathbf{b}} d S^{\prime} \mathbf{G}_{D}\left(x, y \mid x^{\prime}, y_{i}\right) \overleftarrow{W}_{i} \gamma^{i}\left(x^{\prime}\right)
$$

Substituting it in (48) similarly to (16) results in the quadratic action in the graviton

$$
S_{\text {grav }}[\gamma]=-\frac{1}{2} \sum_{i, j} \int_{\mathbf{b}_{i}} d S \int_{\mathbf{b}_{j}} d S^{\prime} \gamma^{i}(x)\left[\overrightarrow{\mathrm{W}} \mathbf{G}_{D} \overleftarrow{W}\right]_{i j}\left(x, x^{\prime}\right) \gamma^{j}\left(x^{\prime}\right)
$$

where

$$
\left[\overrightarrow{\mathrm{W}} \mathbf{G}_{D} \overleftarrow{\mathrm{W}}\right]_{i j}\left(x, x^{\prime}\right)=a_{i}^{d} a_{j}^{d} \nabla_{\mathbf{n}_{i}} \nabla_{\mathbf{n}_{j}} \mathbf{G}_{D}\left(x, y_{i} \mid x^{\prime}, y_{j}\right)
$$

and the primed integration measure is obviously $d S^{\prime}=d^{d} x^{\prime} \sqrt{g\left(x^{\prime}\right)}$.

In what follows the operator notations for the kernels of integral operations will be more convenient for our purposes. This operator form follows from the fact that the differential operator (49) depends on $\square$ as a parameter, therefore the kernel of its inverse can be written down as a function of the $\square$ acting on $d$-dimensional $\delta$-function $\delta^{(d)}\left(x, x^{\prime}\right) \equiv$ $\delta^{d}\left(x-x^{\prime}\right) / \sqrt{g(x)}$

$$
\left[\overrightarrow{\mathrm{W}} \mathbf{G}_{D} \overleftarrow{\mathrm{W}}\right]_{i j}(\square) \delta^{(d)}\left(x, x^{\prime}\right)=\left[\overrightarrow{\mathrm{W}} \mathbf{G}_{D} \overleftarrow{W}\right]_{i j}\left(x, x^{\prime}\right)
$$

\footnotetext{
${ }^{12}$ Note that the kernel of the Green's function remains a biscalar with respect to both of its arguments.
} 
Thus, finally, after combining together tensor and scalar parts and writing explicitly the integration measure, the quadratic part of braneworld effective action looks as follows

$$
S_{d}^{(2)}[h]=\frac{1}{2} \int_{\mathbf{b}} d^{d} x \sqrt{g(x)} \sum_{i, j= \pm}\left(-\gamma^{i}(x)\left[\overrightarrow{\mathrm{W}} \mathbf{G}_{D} \overleftarrow{\mathrm{W}}\right]_{i j}(\square) \gamma^{j}(x)+\psi^{i}(x) \mathrm{K}_{i j}(\square) \psi^{j}(x)\right)
$$

where the notation $\psi^{i}(x)$ stands for $\psi\left(x, y_{i}\right)$ and

$$
\mathrm{K}_{i j}(\square)=4(d-1) \frac{a_{i}^{d-2}}{\nabla_{\mathbf{n}_{i}} \ln a} \delta_{i j}\left(\square+\frac{R(g)}{(d-1)}\right) .
$$

This matrix operator for the case of $d=4$ flat branes coincides via appropriate rescaling with the operator $\mathbf{K}_{\Phi}(\square)$ calculated in [14] by a different procedure.

At this point it is worth discussing why the sector of transverse-traceless modes in (56) can be regarded as the graviton sector even for curved branes. The recovery of a particle interpretation for the effective action with the essentially nonlocal form factor (55) is a matter of the low-energy approximation - the expansion of this form factor in powers of $\square \rightarrow 0$. In this paper we will not consider this expansion in much detail because it was systematically developed in [14] for flat branes. Here we will only sketch a similar procedure and its peculiarities due to curvature of a homogeneous brane background.

To begin with, note that the $d$-dimensional term $\square-2 R(g) / d(d-1)$ of the operator $\hat{\mathbf{F}}$, (49), also enters as a whole the quadratic term $\gamma_{\alpha \beta}(\square-2 R(g) / d(d-1)) \gamma^{\alpha \beta}$ of the $\gamma$-perturbation expansion for the $d$-dimensional Einstein-Hilbert action

$$
S_{E H}[g+\gamma]=\int d^{d} x \sqrt{\operatorname{det}(g+\gamma)}(R(g+\gamma)-2 \lambda)
$$

having a particular value of the cosmological constant

$$
\lambda=\frac{d-2}{2 d} R(g),
$$

which supports the vacuum homogeneous background with a constant scalar curvature $R(g)$. This is the reason why in the low-energy approximation the usual Einstein gravity theory is recovered on a positive tension brane even when it is not flat. The mechanism of this phenomenon is the following. The nonlocal operator (55) of the transverse traceless sector parametrically depends on $\square-2 R(g) / d(d-1)$ as a whole and, therefore, can be expanded in powers of this combination. One can show that this expansion starts with the first order term (for properly defined graviton fields diagonalising the quadratic form in (56), which means that this mode is massless [14, 24]. The masslessness implies that on the maximally symmetric spacetime, $R_{\alpha \beta \mu \nu}(g)=\left(g_{\alpha \mu} g_{\beta \nu}-g_{\alpha \nu} g_{\beta \mu}\right) R(g) / d(d-1)$, the solutions of the linearized equations of motion for transverse-traceless modes

$$
\left(\square-\frac{2 R(g)}{d(d-1)}\right) \gamma_{\alpha \beta}(x)=0
$$

still have a residual gauge freedom $\gamma_{\alpha \beta}(x) \rightarrow \gamma_{\alpha \beta}(x)+2 \nabla_{(\alpha} \xi_{\beta)}(x)$ with the transverse vector field, $\nabla_{\alpha} \xi^{\alpha}=0$, satisfying the equation $2 \nabla^{\beta} \nabla\left(\alpha \xi_{\beta}\right)=(\square+R(g) / d) \xi_{\alpha}=0$. The 
transversality together with this equation guarantee that these transformations preserve transverse-traceless nature of $\gamma_{\alpha \beta}(x)$ and the equations of motion (60) because $(\square-$

$2 R(g) / d(d-1)) \nabla_{(\alpha} \xi_{\beta}=0$. Thus, these residual transformations reduce the number of physical degrees of freedom for $\gamma_{\alpha \beta}$ by $d-1$ to that of the $d$-dimensional massless graviton $d(d-3) / 2$. This justifies the interpretation of the transverse-traceless modes as gravitons.

\section{Alternative derivation of the braneworld effective action}

There exists an alternative derivation of the effective action suggested in [13, 14]. It is based on the recovery of the action from the effective equations for brane $d$-dimensional field. These effective equations actually represent the Israel junction conditions on branes rewritten entirely in terms of the brane metric itself. In order to see this let us write down the metric variation of the fundamental $(d+1)$-dimensional action (20)

$$
\begin{gathered}
\delta \mathbf{S}[G, \varphi]=-\int_{\mathbf{B} \times Z_{2}} d^{d+1} X \sqrt{G}\left(R^{A B}(G)-\frac{1}{2} R(G) G^{A B}+\Lambda G^{A B}\right) \delta G_{A B}(X)+ \\
\quad+\sum_{i} \int_{\mathbf{b}_{i}} d^{d} x \sqrt{g}\left(\left[K^{\mu \nu}-g^{\mu \nu} K\right]+\frac{1}{2}\left(T^{\mu \nu}-g^{\mu \nu} \sigma_{i}\right)\right) \delta g_{\mu \nu}(x) .
\end{gathered}
$$

Here $g_{\mu \nu}(x)$ denotes the induced metric on branes (in contrast with the notations of the previous section, where $g_{\mu \nu}(x)$ was reserved for the auxiliary homogeneous metric), $\left[K^{\mu \nu}-g^{\mu \nu} K\right]$ is the jump of the extrinsic curvature terms across the brane, and $T^{\mu \nu}(x)$ is the corresponding $d$-dimensional stress tensor of matter fields on branes

$$
T^{\mu \nu}(x)=\frac{2}{\sqrt{g}} \frac{\delta S^{\mathrm{mat}}[g, \varphi]}{\delta g_{\mu \nu}(x)} .
$$

Note that the brane part of the (61) gives the Israel junction conditions.

Suppose that we solved Einstein equations of motion in the bulk subject to fixed brane metrics and substituted the obtained solution $G_{A B}=G_{A B}[g]$ in the original action in order to find the effective one, $S^{\text {eff }}[g, \varphi]=\mathbf{S}[G[g], \varphi]$. Then the variation of the latter will not contain the bulk term of (61) - because the bulk equations are satisfied - and the metric variation of $S^{\text {eff }}[g, \varphi]$ will be

$$
\frac{\delta S^{\mathrm{eff}}[g, \varphi]}{\delta g_{\mu \nu}(x)}=\sqrt{g}\left(\left[K^{\mu \nu}-g^{\mu \nu} K\right]+\frac{1}{2}\left(T^{\mu \nu}-g^{\mu \nu} \sigma_{i}\right)\right)_{\mathbf{b}_{i}} .
$$

Here, certainly, the extrinsic curvatures containing the derivatives normal to brane surfaces are the functions of brane metrics. Thus, this equation can be functionally integrated to recover $S^{\text {eff }}[g, \varphi]$. The result (63) is exact, but expressing its right hand side in terms of brane metrics can in general be obtained only within perturbation theory. This procedure was implemented in [14] in the quadratic approximation in metric perturbations on 
a flat brane background. To simplify the presentation we briefly repeat this procedure in our case.

We start with the linearized version of the bulk Einstein equations and linearized Israel matching conditions on the branes. For transverse-traceless perturbations $\gamma_{\alpha \beta}(x, y)$ they reduce to the linear Neumann boundary value problem

$$
\left\{\begin{array}{l}
\hat{\mathbf{F}} \gamma_{\alpha \beta}(x, y)=0 \\
\left.a^{d} \nabla_{\mathbf{n}} \gamma_{\alpha \beta}(x, y)\right|_{\mathbf{b}_{i}}=-\frac{1}{2} t_{\alpha \beta}^{i}(x)
\end{array}\right.
$$

On account of the constraints (46) in the bulk, the vector part of the equations is trivially satisfied, while their scalar part is locally restricted to branes and reads [21]

$$
4(d-1) \frac{a_{i}^{-2}}{\nabla_{\mathbf{n}_{i}} \ln a}\left(\square+\frac{R(g)}{(d-1)}\right) \psi^{i}(x)=-t_{\text {scal }}^{i}(x) .
$$

From now on we go back to notations of the previous section: $\hat{\mathbf{F}}$ is the operator (49) acting in the bulk, $\nabla_{\mathbf{n}}$ is the derivative normal to the brane (28), $t_{\alpha \beta}^{i}(x)$ is the transverse traceless part of the rescaled matter stress tensor $a^{d-2} T_{\alpha \beta}(x)$ on the $i$-th brane and $t_{\text {scal }}^{i}=a^{d-2} g^{\alpha \beta}(x) T_{\alpha \beta}^{i}(x)$ (where indices of $T_{\alpha \beta}^{i}(x)$ were lowered with the induced metric on appropriate brane). Greek indices are raised and lowered with the aid of the auxiliary fixed metric $g_{\alpha \beta}(x)$ of the homogeneous spacetime, which is conformally related to background metrics of all branes (differing only by conformal factors $a_{i}^{2}$ ) which agrees with convention of previous section.

The general solution of Neumann boundary value problem (64) ist3

$$
\gamma(x, y)=-\frac{1}{2} \sum_{i} \int_{\mathbf{b}} d S^{\prime} \mathbf{G}_{N}\left(x, y \mid x^{\prime}, y_{i}\right) t_{i}\left(x^{\prime}\right),
$$

where $\mathbf{G}_{N}\left(x, y, x^{\prime}, y_{i}\right)$ is the Neumann Green's function

$$
\left\{\begin{array}{l}
\hat{\mathbf{F}} \mathbf{G}_{N}\left(x, y \mid x^{\prime}, y^{\prime}\right)=\delta^{(d+1)}\left(x, y \mid x^{\prime}, y^{\prime}\right) \\
\left.\nabla_{\mathbf{n}} \mathbf{G}_{N}\left(x, y \mid x^{\prime}, y^{\prime}\right)\right|_{\mathbf{b}_{i}}=0
\end{array}\right.
$$

Effective dynamics of the brane metric allows one to express the latter solely in terms of brane matter sources. In the transverse-traceless sector this reduces to restricting the general bulk solution to branes, i.e. by putting in (66) $y=y_{j}$. In terms of the operator notation for the kernel of the Neumann Green's function (similar to (55)),

$$
\mathbf{G}_{N}\left(x, y_{j} \mid x^{\prime}, y_{i}\right)=\mathbf{G}_{N}^{j i}(\square) \delta^{(d)}\left(x, x^{\prime}\right)
$$

this reads as

$$
\gamma^{j}(x)=-\frac{1}{2} \sum_{i} \mathbf{G}_{N}^{j i}(\square) t_{i}(x) .
$$

\footnotetext{
${ }^{13} \mathrm{As}$ in the case of the Dirichlet boundary value problem above in what follows we drop for brevity Greek indices in linear and quadratic combinations of $\gamma_{\alpha \beta}(x, y)$ and $t_{\alpha \beta}^{i}(x)$.
} 
There are infinitely many actions that generate by variational procedure the last equation. Fortunately, there is a hint coming from Eq. 63) that allows one to fix this ambiguity - the rescaled matter stress tensor enters the variational derivative of the braneworld action with respect to induced metric $a^{2} g_{\alpha \beta}(x)$ with the algebraic coefficient $a^{-2} \sqrt{g(x)}$. Therefore, by rewriting (69) and (65) as

$$
\begin{aligned}
& \sqrt{g(x)} \sum_{j}\left(\mathbf{G}_{N}^{-1}\right)_{i j}(\square) \gamma^{j}(x)+\frac{1}{2} \sqrt{g(x)} t_{i}(x)=0, \\
& \sqrt{g(x)} \sum_{j} \mathrm{~K}_{i j}(\square) \psi^{j}(x)+\sqrt{g(x)} t_{i}^{\text {scal }}(x)=0,
\end{aligned}
$$

where $\mathrm{K}_{i j}(\square)$ is defined by $(57)$, and $\left(\mathbf{G}_{N}^{-1}\right)_{i j}(\square)$ is the inverse of $\mathbf{G}_{N}^{j i}(\square)$

$$
\sum_{j}\left(\mathbf{G}_{N}^{-1}\right)_{i j}(\square) \mathbf{G}_{N}^{j k}(\square)=\delta_{i}^{k},
$$

one easily finds the action generating (70)

$$
\begin{gathered}
S^{\mathrm{eff}}[h, \varphi]=\frac{1}{2} \int_{\mathbf{b}} d^{d} x \sqrt{g(x)} \sum_{i, j}\left(\gamma^{i}(x)\left(\mathbf{G}_{N}^{-1}\right)_{i j}(\square) \gamma^{j}(x)+\psi^{i}(x) \mathrm{K}_{i j}(\square) \psi^{j}(x)\right) \\
+\frac{1}{2} \int_{\mathbf{b}} d^{d} x \sqrt{g(x)} \sum_{i}\left(\gamma^{i}(x) t_{i}(x)+2 \psi^{i}(x) t_{i}^{\mathrm{scal}}(x)\right) .
\end{gathered}
$$

The source term with $t^{i}(x)$ and its trace here is obviously a linear in metric perturbation part of the action of matter field, while the quadratic in $\gamma$ part has a purely gravitational nature and, thus, should necessarily coincide with the action (56) obtained within the Dirichlet boundary conditions. This implies the equality of two operators arising in two different boundary value problems - the Dirichlet and Neumann one

$$
\left[\overrightarrow{\mathrm{W}} \mathbf{G}_{D} \overleftarrow{\mathrm{W}}\right]_{i j}(\square)=-\left(\mathbf{G}_{N}^{-1}\right)_{i j}(\square)
$$

\section{$5 \quad$ Duality of boundary value problems}

To prove the duality relation (73) let us, first, remind the notations of Sect.2. We consider $\mathcal{M}$ - the bulk spacetime with branes/boundaries $\partial \mathcal{M}_{i}: \partial \mathcal{M}=\bigcup_{i} \partial \mathcal{M}_{i}$. Let $X$ be the coordinates on the bulk $\mathcal{M}$ and $x$ - the coordinates on the branes $\partial \mathcal{M}_{i}$, so that the embedding of $\partial \mathcal{M}_{i}$ into $\mathcal{M}$ reads as $X=X_{i}(x)$. Introduce $H$ - the field in the bulk and denote boundary values of the bulk field on $\partial \mathcal{M}_{i}$ by $h^{i}, h^{i}(x)=H\left(X_{i}(x)\right)$. Let $\hat{\mathbf{F}}$ be some nondegenerate self-adjoint differential operator of the second order in derivatives acting on $H(X)$. Let it satisfy the Wronskian relation (10) with the first order Wronskian operator $\hat{W}$.

Let $H(X)$ satisfy the Neumann problem with the inhomogeneous boundary conditions with some given sources $j_{k}(x)$ on the boundary

$$
\left\{\begin{array}{l}
\hat{\mathbf{F}} H(X)=0 ; \\
\left.\hat{W} H(X)\right|_{X=X_{k}(x)}=j_{k}(x) .
\end{array}\right.
$$


The solution of this problem in terms of the Neumann Green's function of the operator $\hat{\mathbf{F}}$, satisfying

$$
\left\{\begin{array}{l}
\hat{\mathbf{F}} \mathbf{G}_{N}\left(X, X^{\prime}\right)=\delta\left(X, X^{\prime}\right) \\
\left.\hat{W} \mathbf{G}_{N}\left(X, X^{\prime}\right)\right|_{X \in \partial \mathcal{M}_{k}}=0
\end{array}\right.
$$

reads

$$
H(X)=\sum_{k} \int_{\partial \mathcal{M}_{k}} d S^{\prime} \mathbf{G}_{N}\left(X, X_{k}\left(x^{\prime}\right)\right) j_{k}\left(x^{\prime}\right) .
$$

Its restriction to the boundary yields the relation between the boundary fields $h^{l}(x)$ and their conjugates - sources $j_{k}(x)$

$$
h^{l}(x)=\sum_{k} \int_{\partial \mathcal{M}_{k}} d S^{\prime} \mathbf{G}_{N}^{l k}\left(x, x^{\prime}\right) j_{k}\left(x^{\prime}\right)
$$

in terms of the new boundary operator kernel

$$
\mathbf{G}_{N}^{l k}\left(x, x^{\prime}\right)=\mathbf{G}_{N}\left(X_{l}(x), X_{k}\left(x^{\prime}\right)\right)
$$

On the other hand the same field (76) serves as the solution of the Dirichlet problem (8) with the boundary data $\left.H(X)\right|_{X=X(x)}=h(x)$ related to the sources $j(x)$ according to (77). Therefore, in terms of the Dirichlet Green's function (12) $H(X)$ has the form (13). Acting on (13) on the boundaries by the Wronskian and taking into account that $\left.\hat{W} H(X)\right|_{\partial \mathcal{M}_{l}}=j_{l}(x)$ one finally obtains the alternative to (77) relation between $h(x)$ and $j(x)$

$$
j_{k}(x)=-\sum_{l} \int_{\partial \mathcal{M}_{l}} d S^{\prime}\left[\vec{W} \mathbf{G}_{D} \overleftarrow{W}\right]_{k l}\left(x, x^{\prime}\right) h^{l}\left(x^{\prime}\right)
$$

where the kernel $\left[\vec{W} \mathbf{G}_{D} \overleftarrow{W}\right]_{k l}\left(x, x^{\prime}\right)$ is defined by Eq.(19)

Comparing (77) and (779) one concludes that $\mathbf{G}_{N}^{l k}\left(x, x^{\prime}\right)$ and $-\left[\vec{W} \mathbf{G}_{D} \overleftarrow{W}\right]_{k l}\left(x, x^{\prime}\right)$ are the kernels of the inverse operations on $\partial \mathcal{M}$

$$
-\sum_{i} \int_{\partial \mathcal{M}_{i}} d S^{\prime}\left[\vec{W} \mathbf{G}_{D} \overleftarrow{W}\right]_{l i}\left(x, x^{\prime \prime}\right) \mathbf{G}_{N}^{i k}\left(x, x^{\prime}\right)=\delta_{k}^{l} \delta\left(x, x^{\prime}\right)
$$

This confirms the operator relation (73). Below we consider two examples demonstrating the efficiency of this result.

\footnotetext{
${ }^{14}$ We define the delta-function $\delta\left(X, X^{\prime}\right)$ by the relation $\int_{\mathcal{M}} d V H(X) \delta\left(X, X^{\prime}\right)=H\left(X^{\prime}\right)$.
} 


\subsection{Simple example}

Consider the simplest model with the operator on the one-dimensional space of the coordinate $y, y_{1} \leq y \leq y_{2}$,

$$
\hat{\mathbf{F}}=\frac{\partial^{2}}{\partial y^{2}}+m^{2}
$$

and introduce its Wronskian operator acting on boundaries

$$
\nabla_{\mathbf{n}}= \begin{cases}+\partial_{y}, & y=y_{1} \\ -\partial_{y}, & y=y_{2}\end{cases}
$$

The Dirichlet and Neumann Green's functions of this operator read

$$
\begin{aligned}
& \mathbf{G}_{D}\left(y, y^{\prime}\right)=\frac{\sin m\left(y^{\prime}-y_{2}\right) \sin m\left(y-y_{1}\right)}{m \sin m\left(y_{2}-y_{1}\right)} \theta\left(y^{\prime}-y\right)+\left(y \leftrightarrow y^{\prime}\right), \\
& \mathbf{G}_{N}\left(y, y^{\prime}\right)=\frac{\cos m\left(y^{\prime}-y_{2}\right) \cos m\left(y-y_{1}\right)}{m \sin m\left(y_{2}-y_{1}\right)} \theta\left(y^{\prime}-y\right)+\left(y \leftrightarrow y^{\prime}\right),
\end{aligned}
$$

so that the operators (19) and (78) turn out to be $2 \times 2$-matrices

$$
\begin{aligned}
& -\left[\vec{\nabla}_{\mathbf{n}} \mathbf{G}_{D} \overleftarrow{\nabla}_{\mathbf{n}}\right]_{i j}=\frac{m}{\sin m\left(y_{2}-y_{1}\right)}\left(\begin{array}{cc}
-\cos m\left(y_{2}-y_{1}\right) & 1 \\
1 & -\cos m\left(y_{2}-y_{1}\right)
\end{array}\right), \\
& \mathbf{G}_{N}^{i j}=\frac{1}{m \sin m\left(y_{2}-y_{1}\right)}\left(\begin{array}{cc}
\cos m\left(y_{2}-y_{1}\right) & 1 \\
1 & \cos m\left(y_{2}-y_{1}\right)
\end{array}\right) .
\end{aligned}
$$

This is a matter of direct verification that these matrices are inverse to one another, because their determinants equal

$$
\operatorname{det} \mathbf{G}_{N}{ }^{i j}=\left(\operatorname{det}\left[\overrightarrow{\mathrm{W}} \mathbf{G}_{D} \overleftarrow{W}\right]_{i j}\right)^{-1}=-\frac{1}{m^{2}}
$$

\subsection{Example: two-brane Randall-Sundrum model}

In this section we illustrate the relation $(\overline{73})$ for the Randall-Sundrum model with two flat branes. As in Sect.3 we work in coordinate system $X^{A}=\left(x^{\alpha}, y\right)$ in which background metric has the form (21) with flat spacetime metric $g_{\alpha \beta}(x)=\eta_{\alpha \beta}$. Two flat branes with tensions $\sigma_{+}=-\sigma_{-}$are located respectively at $y=y_{+}$and $y=y_{-}$. In such a coordinate system

$$
\begin{aligned}
& \hat{\mathbf{F}}=a^{-d} \partial_{y} a^{d} \partial_{y}+a^{-2} \square ; \\
& \eta^{\alpha \beta}(x) \eta_{\alpha \beta}(x)=d, \quad \square=\eta^{\alpha \beta} \nabla_{\alpha} \nabla \beta, \\
& \nabla_{\mathbf{n}} \equiv \mathbf{n}^{A} \nabla_{A}= \begin{cases}+\partial_{y}, & y=y_{+}, \\
-\partial_{y}, & y=y_{-} .\end{cases}
\end{aligned}
$$


For the sake of brevity we introduce the new operator-valued variable depending on the coordinate $y$

$$
\begin{aligned}
& \varkappa \equiv \varkappa(y, \square)=\frac{\sqrt{\square}}{k a(y)}, \\
& \varkappa_{+}=\varkappa\left(y_{+}, \square\right), \quad \varkappa_{-}=\varkappa\left(y_{-}, \square\right),
\end{aligned}
$$

and the following two-point combinations of Bessel functions

$$
\begin{aligned}
& \mathbf{D}\left(\varkappa, \varkappa^{\prime}\right)=\frac{\pi}{2}\left(Y_{d / 2}(\varkappa) J_{d / 2}\left(\varkappa^{\prime}\right)-Y_{d / 2}\left(\varkappa^{\prime}\right) J_{d / 2}(\varkappa)\right), \\
& \mathbf{E}\left(\varkappa, \varkappa^{\prime}\right)=\frac{\pi}{2}\left(Y_{d / 2}(\varkappa) J_{d / 2-1}\left(\varkappa^{\prime}\right)-Y_{d / 2-1}\left(\varkappa^{\prime}\right) J_{d / 2}(\varkappa)\right), \\
& \mathbf{C}\left(\varkappa, \varkappa^{\prime}\right)=\frac{\pi}{2}\left(Y_{d / 2-1}(\varkappa) J_{d / 2-1}\left(\varkappa^{\prime}\right)-Y_{d / 2-1}\left(\varkappa^{\prime}\right) J_{d / 2-1}(\varkappa)\right) .
\end{aligned}
$$

Then the explicit expressions for Dirichlet and Neumann Green functions take the form 14

$$
\begin{aligned}
& \mathbf{G}_{D}\left(y, y^{\prime} \mid \square\right)=\frac{\mathbf{D}\left(\varkappa^{\prime}, \varkappa_{-}\right) \mathbf{D}\left(\varkappa, \varkappa_{+}\right) \theta\left(y^{\prime}-y\right)+\mathbf{D}\left(\varkappa^{\prime}, \varkappa_{+}\right) \mathbf{D}\left(\varkappa, \varkappa_{-}\right) \theta\left(y-y^{\prime}\right)}{a(y)^{d / 2} a\left(y^{\prime}\right)^{d / 2} k \mathbf{D}\left(\varkappa_{-}, \varkappa_{+}\right)}, \\
& \mathbf{G}_{N}\left(y, y^{\prime} \mid \square\right)=\frac{\mathbf{E}\left(\varkappa^{\prime}, \varkappa_{-}\right) \mathbf{E}\left(\varkappa, \varkappa_{+}\right) \theta\left(y^{\prime}-y\right)+\mathbf{E}\left(\varkappa^{\prime}, \varkappa_{+}\right) \mathbf{E}\left(\varkappa, \varkappa_{-}\right) \theta\left(y-y^{\prime}\right)}{a(y)^{d / 2} a\left(y^{\prime}\right)^{d / 2} k \mathbf{C}\left(\varkappa_{-}, \varkappa_{+}\right)} .
\end{aligned}
$$

After acting on the Dirichlet Green's function with normal derivatives (89) and restricting them to branes $\left(y, y^{\prime}=y_{+}, y_{-}\right)$one obtains the $2 \times 2$-matrix valued operators the kernel of the braneworld effective action in the Dirichlet setup (56),

$$
-\left[\overrightarrow{\mathrm{W}} \mathbf{G}_{D} \overleftarrow{\mathrm{W}}\right]_{i j}(\square)=\frac{k}{\mathbf{D}\left(\varkappa_{-}, \varkappa_{+}\right)}\left(\begin{array}{cc}
a_{+}^{d} \varkappa_{+} \mathbf{E}\left(\varkappa_{-}, \varkappa_{+}\right) & a_{+}^{d / 2} a_{-}^{d / 2} \\
a_{+}^{d / 2} a_{-}^{d / 2} & a_{-}^{d} \varkappa_{-} \mathbf{E}\left(\varkappa_{+}, \varkappa_{-}\right)
\end{array}\right)
$$

and the inverse of the kernel in the Neumann setting (72)

$$
\mathbf{G}_{N}^{i j}(\square)=\frac{\left(k \varkappa_{+} \varkappa_{-}\right)^{-1}}{\mathbf{C}\left(\varkappa_{-}, \varkappa_{+}\right)}\left(\begin{array}{cc}
-a_{+}^{-d} \varkappa_{-} \mathbf{E}\left(\varkappa_{+}, \varkappa_{-}\right) & a_{+}^{-d / 2} a_{-}^{-d / 2} \\
a_{+}^{-d / 2} a_{-}^{-d / 2} & -a_{-}^{-d} \varkappa_{+} \mathbf{E}\left(\varkappa_{-}, \varkappa_{+}\right)
\end{array}\right) .
$$

Here we used the properties of Bessel functions [25]

$$
\partial_{\varkappa}\left(\varkappa^{d / 2} \mathbf{D}\left(\varkappa^{\prime}, \varkappa\right)\right)=\varkappa^{d / 2} \mathbf{E}\left(\varkappa^{\prime}, \varkappa\right), \quad \mathbf{E}(\varkappa, \varkappa)=-\varkappa^{-1} .
$$

The calculation of the $2 \times 2$-determinants of these matrices can be easily done by using a simple relation

$$
\mathbf{E}\left(\varkappa^{\prime}, \varkappa^{\prime}\right) \mathbf{E}(\varkappa, \varkappa)-\mathbf{E}\left(\varkappa^{\prime}, \varkappa\right) \mathbf{E}\left(\varkappa, \varkappa^{\prime}\right)=\mathbf{D}\left(\varkappa, \varkappa^{\prime}\right) \mathbf{C}\left(\varkappa, \varkappa^{\prime}\right)
$$

from which it follows that

$$
\operatorname{det} \mathbf{G}_{N}^{i j}(\square)=\left(\operatorname{det}\left[\overrightarrow{\mathrm{W}} \mathbf{G}_{D} \overleftarrow{W}\right]_{i j}(\square)\right)^{-1}=-\frac{\left(k^{2} \varkappa_{-} \varkappa_{+}\right)^{-1}}{a_{+}^{d} a_{-}^{d}} \frac{\mathbf{D}\left(\varkappa_{-}, \varkappa_{+}\right)}{\mathbf{C}\left(\varkappa_{-}, \varkappa_{+}\right)}
$$

Then it is a matter of direct check that the matrices (94) and (95) are inverse to one another. 


\section{Conclusions}

Thus we obtained the braneworld effective action in the weak field (quadratic) approximation for models of curved branes with the deSitter and Anti-deSitter geometries, embedded in the Anti-deSitter bulk. The calculation was done within two different schemes resorting to Dirichlet and Neumann boundary value problems, and their equivalence was shown on the basis of a special relation between the boundary operators associated respectively with the Dirichlet and Neumann Green's functions of the theory. Although this relation was used here only in a braneworld context, it seems to have a much wider scope of implications in various models relating volume (bulk) and surface phenomena, like graviton localization [12], AdS/CFT correspondence and holography principle [26, 27, 28, 29, 30].

Regarding the braneworld action algorithms for curved branes - this result can be important for the brane models of inflation scenario [11, 13, 14. One of the motivations for considering deSitter type branes is that they model the inflation stage in the dynamics of the brane Universe embedded in the multidimensional Anti-deSitter bulk. The idea of the radion field playing the role of the inflaton was suggested in [13], where inflation was induced by the effectively repulsive force between the branes diverging in the course of inflationary stage. This repulsive force arises in virtue of the detuning of brane tensions from their Randall-Sundrum values (26) corresponding to flat branes. However, the analysis of this detuning in [13, 14] was done by directly extrapolating the flat branes results to curved branes. Now, with the knowledge of the answer (56) the effects of curvature can be studied at a rigorous quantitative level. This and, in particular, the effects of brane curvature on the low-energy limit of the nonlocal form factor (54)-(55) will be considered elsewhere.

Another possible generalization of the obtained results concerns going beyond the limitation of conformally equivalent branes. Note that everywhere in the constructions above both branes are "parallel" surfaces simultaneously of the flat, deSitter or AntideSitter geometry. It would be interesting to generalize calculations to coexisting branes of different types (different signs of curvature), because this might lead to the dynamical description of intersecting curved branes very popular in recent years [6, 31, 32, 33, 34]. Finally, the algorithm (56) suggests a natural generalization to multibrane cases when the range of index $i$ goes beyond two possible values. This generalization particularly implies that a brane can serve as a boundary of more than two smooth bulks glued to it. Such a construction of branching bulks can be shown to have explicit Randall-Sundrum type solutions of Einstein equations with appropriately generalized Israel junction conditions 35. It forms a kind of spacetime bulk-brane foam resembling selfreproducing inflationary Universe [36] and suggesting new interesting facets in the cosmological constant problem.

\section{Acknowledgements}

The work of A.O.B was supported by the RFBR grant No 02-01-00930. The work of D.V.N. was supported by the RFBR grant No 02-02-17054 and by the Landau Foundation. This work was also supported by the RFBR grant No 00-15-96566. 


\section{References}

[1] I.Antoniadis, Phys. Lett. B 246 (1990) 377.

[2] J.Polchinski, Phys. Rev. Lett. 75 (1995) 4724, hep-th/9510017; P.Hořava and E.Witten, Nucl. Phys. B 460 (1996) 506, [hep-th/9510209.

[3] N.Arkani-Hamed, S.Dimopoulos and G.Dvali, Phys. Lett. B 429 (1998) 263, [hepph/9803315].

[4] W.Israel, Nuovo Cim. 44S10 (1966) 1.

[5] J.Garriga and M.Sasaki, Phys. Rev. D62 (2000) 043523, hep-th/9912118.

[6] H.A.Chamblin and H.S.Reall, Nucl. Phys. B 562 (1999) 133, hep-th/9903225.

[7] S.W.Hawking, T.Hertog and H.S.Reall, Phys. Rev. D62 (2000) 043501, [hepth/0003052; Phys. Rev. D63 (2001) 083504 , hep-th/0010232.

[8] M.K.Parikh and S.N.Solodukhin, Phys. Lett. B 503 (2001) 384-393, hep-th/0012231.

[9] U.Gen and M.Sasaki, Prog. Theor. Phys. 105 (2001) 591, gr-qc/0011078.

[10] J.Garriga, O.Pujolas and T.Tanaka, "Moduli effective action in warped brane-world compactifications", hep-th/0111277.

[11] G.Dvali and S.-H.H.Tye, Phys. Lett. B 450 (1999) 72.

[12] L.Randall and R.Sundrum, Phys. Rev. Lett. 83 (1999) 4690, hep-th/9906064; Phys. Rev. Lett. 83 (1999) 3370, hep-th/9905221.

[13] A.O.Barvinsky, Phys. Rev. D65 (2002) 062003, hep-th/0107244.

[14] A.O.Barvinsky, A.Yu.Kamenshchik, C.Kiefer and A.Rathke, "Braneworld effective action: An alternative to Kaluza-Klein reduction", hep-th/0206188.

[15] V.F.Mukhanov, H.A.Feldmann and R.H.Brandenberger, Phys. Rep. 115 (1992) 203.

[16] J.Garriga, X.Montes, M.Sasaki and T.Tanaka, Nucl. Phys. B 513 (1998) 343-374, astro-ph/9706229.

[17] A.O.Barvinsky and D.V.Nesterov, Nucl. Phys. B 608 (2001) 333-374, gr-qc/0008062].

[18] E.E.Boos, Y.A.Kubyshin, M.N.Smolyakov and I.P.Volobuev, "Effective Lagrangians for linearized gravity in Randall-Sundrum model", [hep-th/0105304].

[19] Y.A.Kubyshin, "Models with extra dimensions and their phenomenology", [hepph/0111027.

[20] A.O.Barvinsky, Phys. Rev. D50 (1994) 5115-5125, gr-qc/9311023. 
[21] J.Garriga and T.Tanaka, Phys. Rev. Lett. 84 (2000) 2778-2781, hep-th/9911055.

[22] C.Charmousis, R.Gregory and V.A.Rubakov, Phys. Rev. D62 (2000) 067505, hepth/9912160].

[23] E.Flanagan, N.Jones, H.Stoica, H.Tye and I.Wasserman, Phys. Rev. D64 (2001) 045007, hep-th/0012129.

[24] A.O.Barvinsky and D.V.Nesterov, "Braneworld effective action for generalized Randall-Sundrum two-brane model", in preparation.

[25] M.Abramowitz and I.Stegun, Handbook of Mathimatical Functions.

[26] V.Balasubramanian, P.Kraus and A.E.Lawrence, Phys. Rev. D59 (1999) 046003, [hep-th/9805171].

[27] S.S.Gubser, Phys. Rev. D63 (2001) 084017, hep-th/9912001.

[28] N.Arkani-Hamed, M.Porrati and L.Randall, "Holography and phenomenology", hepth/0012148].

[29] M.Henningson and K.Skenderis, Fortsch. Phys. 48 (2000) 125, hep-th/9812032; K.Skenderis and S.N.Solodukhin, Phys. Lett. B 472 (2000) 316, hep-th/9910023.

[30] I.Sachs and S.N.Solodukhin, Phys. Rev. D64 (2001) 124023, hep-th/0107173.

[31] J.Khoury, B.A.Ovrut, P.J.Steinhardt and N.Turok, Phys. Rev. D64 (2001) 123522, hep-th/0103239; P.J.Steinhardt and N.Turok, "A cyclic model of the universe", arXiv:hep-th/0111030]; Phys. Rev. D65 (2002) 126003, hep-th/0111098].

[32] R.Kallosh, L.Kofman and A.D.Linde, Phys. Rev. D64 (2001) 123523, hepth/0104073].

[33] A.Neronov, "Brane collisions in anti-de Sitter space", hep-th/0109090].

[34] M.Bucher and C.Carvalho, "Separation Distribution of Vacuum Bubbles in de Sitter Space", [hep-ph/0207275]; M.Bucher and J.Blanco-Pillado, Phys. Rev. D65 (2002) 083517, hep-th/0111089.

[35] D.V.Nesterov, work in progress.

[36] A.D.Linde, "Particle Physics and Inflationary Cosmology", Harwood Academic, 1990. 